mediated reaction whereby $\operatorname{IgG}$ and $\operatorname{IgM}$ antibodies are directed against heparin and platelet factor. ${ }^{4}$ Desensitization is not a therapeutic option for acute or delayed HIT. Cases have been reported in which alternative drugs, such as bovine heparin, ancrod, and argatroban, have been used in place of heparin when anaphylactoid reactions are suspected; however, their efficacy has been anecdotal and needs further investigation. ${ }^{4}$

Two reports exist in the literature in which desensitization for heparin allergy was attempted before cardiopulmonary bypass. ${ }^{2,5}$ Until more experience is gained with the use of alternative anticoagulation agents for cardiopulmonary bypass, we advocate the use of heparin desensitization as successfully performed in this case.

\section{References}

1. Bernstein IL. Anaphylaxis to heparin sodium; report of a case, with immunologic studies. JAMA. 1956;161:1379-81.

2. al-Eryani AY, al-Momen AK, Fayed DF, Allam AK. Successful heparin desensitization after heparin-induced anaphylactic shock. Thromb Res. 1995;79:523-6.

3. Bottio T, Pittarello G, Bonato R, Fagiolo U, Gerosa G. Life-threatening anaphylactic shock caused by porcine heparin intravenous infusion during mitral valve repair. J Thorac Cardiovasc Surg. 2003;126:1194-5.

4. Smith RE, Townsend GE, Berry BR, Bowen T. Enoxaparin for unstable angina and ancrod for cardiac surgery following heparin allergy. Ann Pharmacother. 1996;30:476-80.

5. Patriarca G, Rossi M, Schiavino D, et al. Rush desensitization in heparin hypersensitivity: a case report. Allergy. 1994;49:292-4.

\title{
Surgical treatment of recurrent transient ischemic attacks and hemoptysis in a young man with multiple pulmonary arteriovenous malformations
}

\author{
Cliff K. Choong, FRACS, ${ }^{a}$ Daniel M. Goodenberger, MD, ${ }^{\mathrm{b}, \mathrm{c}}$ Daniel Picus, MD, ${ }^{\mathrm{b}, \mathrm{d}}$ and \\ Bryan F. Meyers, MD, ${ }^{\text {a }}$ St Louis, Mo
}

$\mathrm{P}$ ulmonary arteriovenous malformation (PAVM) is a rare vascular anomaly with an incidence of 2 to 3 per 100,000 population. ${ }^{1}$ We present a patient whose therapy with embolotherapy for PAVMs resulted in recurrent transient ischemic attacks (TIAs) and massive hemoptysis requiring treatment by surgical resection.

\section{Clinical Summary}

A 17-year-old man with hereditary hemorrhagic telangiectasia (HHT) had PAVMs incidentally discovered on a chest $\mathrm{x}$-ray film. Investigations confirmed PAVMs without the presence of associated bronchial collateral artery. Although his pulse oxygen saturation at rest was $80 \%$, he was asymptomatic. He underwent embolization therapy with aneurysmal coil placement and cyanoacrylate glue elsewhere (Figures 1A and 1B). One week later, he had the first of recurrent TIAs with tingling and numbness in the

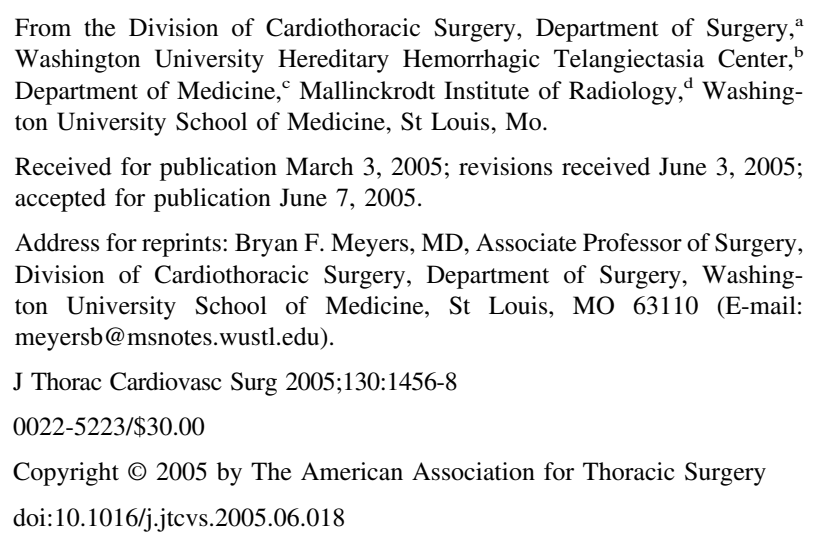

right upper extremity. Investigations including carotid duplex scan, echocardiography, and cerebral CT and MRI did

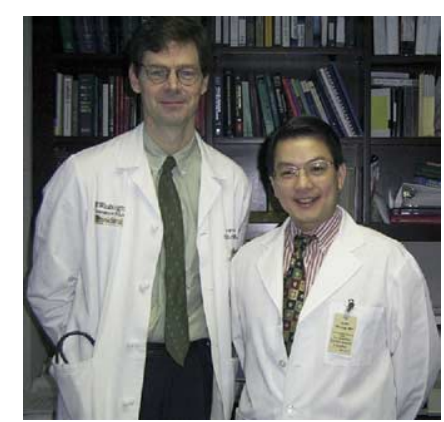

Drs Meyers and Choong not reveal any abnormalities. The TIAs were considered to be most likely caused by emboli originating from clot propagation distal to the coils. Six months later, he had the first of multiple episodes of hemoptysis of 16 ounces or more, which were unresponsive to embolotherapy of several smaller PAVMs. Angiography at our center showed occlusion of the majority of the PAVMs. A large bronchial artery was however seen, supplying an area of neovascularization of the inflammatory complex of cyanoacrylate in the apical segment of the right lower lobe, with rapid filling of the pulmonary vein, implying arteriovenous shunting (Figure 2A). The vessel was larger than the largest Ivalon particles, and an attempt to occlude it by embolotherapy was considered to be potentially hazardous and unlikely to result in a lasting occlusive effect. A decision was made to remove the large PAVMs of the right lower lobe by surgical resection. He underwent an uneventful right lower lobectomy and at 1-year follow-up, he remained well with no further TIA or hemoptysis. A transverse section of the resected lung with coils in the PAVM is shown in Figure 2B.

\section{Discussion}

PAVM was first reported in 1897 by Churton in a 12-year-old boy who had episodes of epistaxis, hemoptysis, and loud pulmonary systolic bruit. ${ }^{2}$ At postmortem examination, he was found to have multiple bilateral PAVMs. More than $80 \%$ of PAVMs are congenital in origin, and of these $60 \%$ to $90 \%$ are associated with HHT. ${ }^{1}$ Conversely, $15 \%$ to $35 \%$ of the population with HHT have a PAVM. The rate of PAVM in our 292 HHT patients was $58 \%$. 


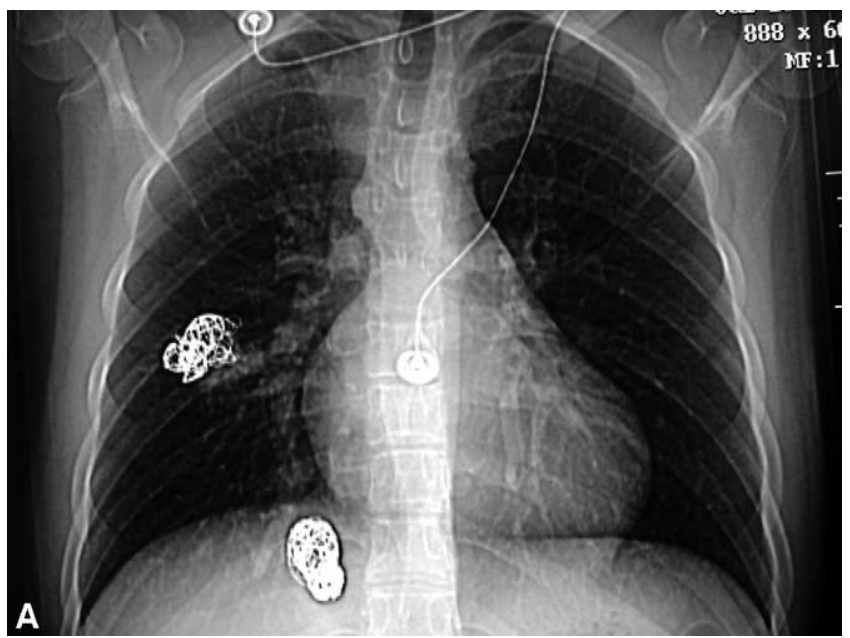

Figure 1A. Chest $x$-ray film showing 2 large PAVMs in the apical and posterior basal segments of the right lower lobe filled with numerous long coils.

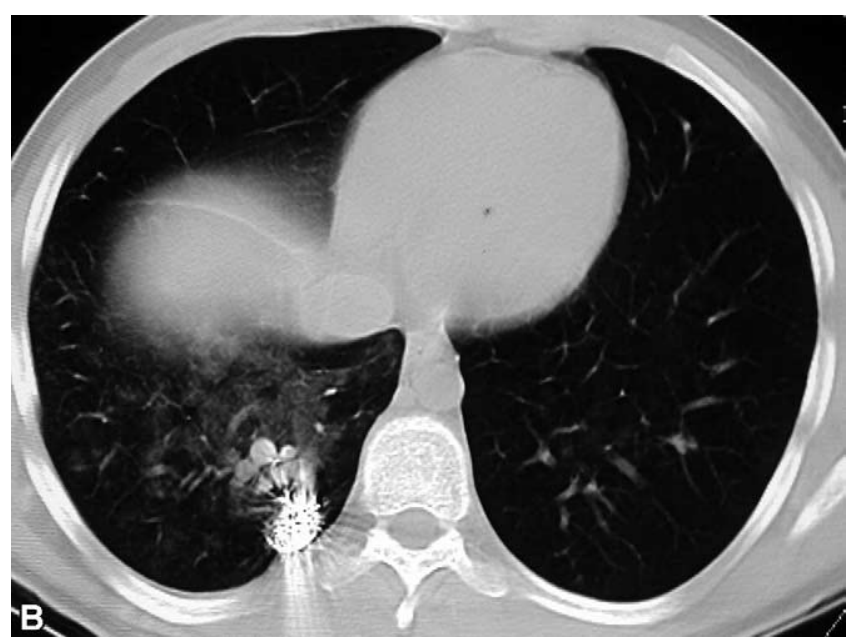

Figure 1B. Chest CT showing coils in the PAVM in the posterior basal segment of the right lower lobe.

The majority of the patients with PAVMs are asymptomatic. The clinical presentations range from an incidental finding on a chest radiograph to hemoptysis, hemothorax, polycythemia, cyanosis, and neurologic deficits secondary to paradoxical embolism. In our experience over a 15-year period, the majority of the patients with PAVMs are asymptomatic with neurologic complications being the most common clinical presentations in the forms of paradoxical embolic stroke (30\%) and brain abscess (5\%). Hemoptysis, hemothorax, polycythemia, and cyanosis are all uncommon. The presence of HHT in a patient with a PAVM is of prognostic value since the patient with coexisting HHT tends to have worse symptomatology, multiple arteriovenous malforma-

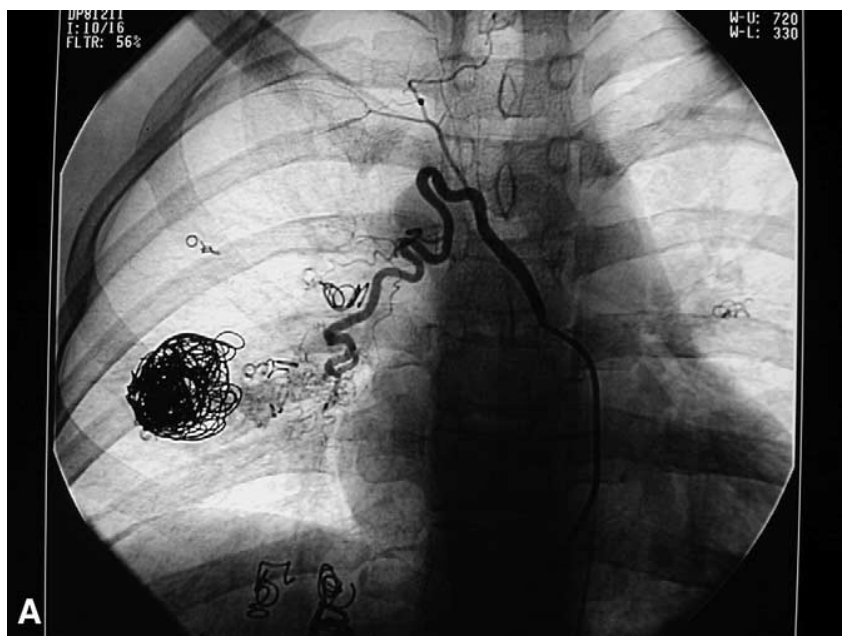

Figure 2A. Angiography showing a large bronchial artery $(8 \mathrm{~mm}$ in size) supplying the PAVM in the apical segment of the right lower lobe and then rapidly filling the pulmonary vein.

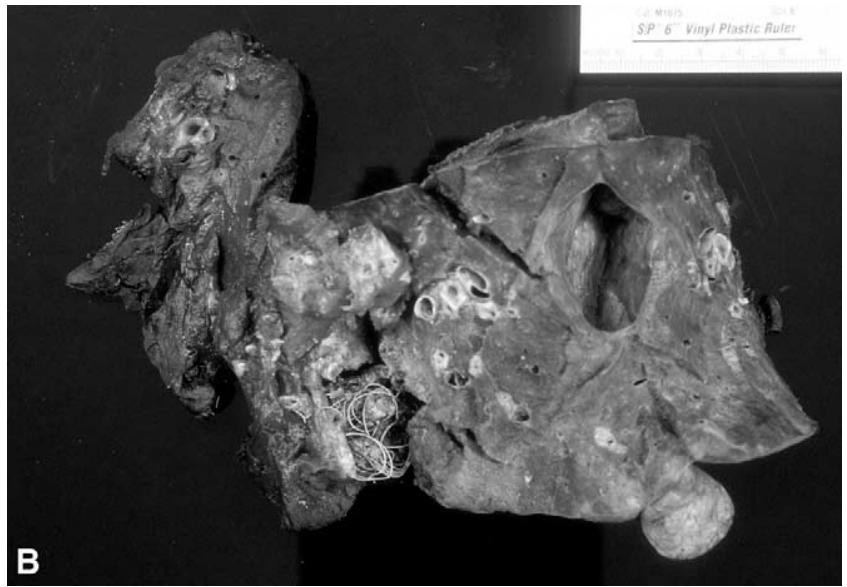

Figure 2B. Cut section of resected right lower lobe showing multiple coils deposited within a large PAVM.

tions, rapid disease progression, and a higher incidence of complication rate. $^{1,3}$

PAVMs may result in serious complications, which can be prevented with appropriate treatment. In 1942, Hepburn and Dauphinee $^{4}$ reported the first case of successful surgical removal of a PAVM with disappearance of the patient's polycythemia and clubbing after a pneumonectomy.

Over the decades, surgical treatments of PAVMs were further refined to ligation of the pulmonary artery, lobectomy, segmentectomy, and local excision. Surgery remained the mainstay of treatment until 1978 when Taylor and coworkers ${ }^{5}$ reported the first case of successful percutaneous catheterization and embolization of a PAVM. Embolotherapy has revolution- 
ized the treatment of PAVMs by virtually eliminating the need for surgery and has the advantages of being less invasive, easy to repeat, and effective. ${ }^{1}$ In a review of 808 embolotherapy attempts for 803 PAVMs in 288 patients, the overall success rate was $99 \%$ with no reported mortality. ${ }^{1}$ The most commonly encountered complication was self-limiting pleuritic chest pain, which was seen in up to $13 \%$ of patients. Other reported complications have included pulmonary infarction, air embolism, device migration, myocardial rupture, cerebrovascular accident, vascular injury, deep vein thrombosis, and pulmonary hypertension. In our experience of 15 years, apart from pleuritic chest pain, we only had a single case of balloon migration to a non-feeding artery and no other complication was encountered. These excellent results, however, depend upon appropriate technique, which involves embolization of the feeding vessel and not the aneurysmal sac, as was done in this case. Cyanoacrylate glue is not indicated for the treatment of PAVMs as there is a significant risk of nontarget embolization by using a liquid agent in a right-to-left shunt. In addition, the tissue infarction is very extensive with cyanoacrylate glue and that likely resulted in the recruitment of bronchial collaterals which led to the life-threatening hemoptysis in this patient. Embolization in PAVMs should be limited to the abnormal shunt and rarely, if ever, should result in tissue damage extensive enough to drive bronchial collateral formation. Surgical resection of PAVM remains an important option and is indicated in patients who cannot be embolized, and we have encountered only 3 such patients over a 15-year period, or those who develop a complication of embolotherapy such as this reported case. In summary, we present a young patient with large PAVMs in the right lower lobe whose inappropriate embolotherapy was complicated by recurrent TIAs and severe hemoptysis. Surgical resection was required and resulted in a successful treatment.

\section{References}

1. Khurshid I, Downie GH. Pulmonary arteriovenous malformation. Postgrad Med J. 2002;78:191-7.

2. Churton T. Multiple aneurysms of pulmonary artery. Br Med J. 1897; $1: 1223$.

3. Guttmacher AE, Marchuk DA, White RI. Hereditary hemorrhagic telangiectasia. N Engl J Med. 1995;333:918-24.

4. Hepburn J, Dauphinee JA. Successful removal of hemangioma of lung followed by disappearance of polycythemia. Am J Med Sci. 1942;204:681-7.

5. Taylor BG, Codkerill EM, Manfredi F, et al. Therapeutic embolization of the pulmonary artery in pulmonary arteriovenous fistula. Am J Med. 1978;64:360-5. 